\title{
Relación entre actividad fisica, sedentarismo y exceso de peso en adolescentes de los Santanderes, Colombia
}

\section{Relationship between physical activity, sedentary lifestyle, and excess weight in teenagers in Santanderes, Colombia}

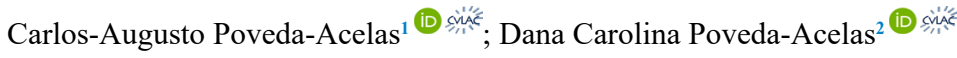

Forma de citar: Poveda-Acelas CA, Poveda-Acelas DC. Relación entre actividad física, sedentarismo y exceso de peso en adolescentes de los Santanderes Colombia. Salud UIS. 2021; e21024. doi: https://doi.org/10.18273/saluduis.53.e:21024 (c) (1)

\section{Resumen}

Introducción: el exceso de peso en adolescentes es uno de los problemas sanitarios más frecuentes a nivel mundial; este es atribuido a factores sociales, familiares y a los estilos de vida, los cuales si no se modifican pueden generar repercusiones físicas, sociales, psicológicas y económicas. Objetivo: determinar la relación entre factores sociodemográficos, actividad física, comportamientos sedentarios y exceso de peso de los adolescentes (13 a 17 años) de la región los Santanderes, Colombia. Materiales y métodos: estudio analítico de corte transversal, de fuente secundaria, proveniente de datos (847 registros) de la Encuesta Nacional de la Situación Nutricional en Colombia (Ensin 2015) sobre la actividad física, sedentarismo y su relación con el exceso de peso en adolescentes de la región de los Santanderes, Colombia. El estudio implicó la realización de la prueba de hipótesis de chi cuadrado con el cálculo de OR como medida epidemiológica con sus respectivos intervalos de confianza al $95 \%$. El análisis multivariado consistió en realizar un modelo de regresión logística binaria con fines explicativos. Resultados: no se encontró relación entre las variables sociales, familiares, de autopercepción corporal, actividad física y comportamientos sedentarios con el exceso de peso; sin embargo, el área de residencia, particularmente vivir en las cabeceras municipales (OR Crudo: 1,635 , IC95 \%: 1,050 a 2,546) y autopercibirse con peso corporal como normal (OR Ajustado: 3,661, IC95 \%: 1,781 a 7,526) aumentan las posibilidades de padecer sobrepeso y obesidad. Conclusión: se requiere la implementación de programas educativos interdisciplinarios que permitan prevenir y tratar oportunamente el exceso de peso en los adolescentes, teniendo en cuenta que existen factores que muestran cierta relación con el exceso de peso como lo son vivir en cabeceras municipales, nivel de escolaridad del jefe de hogar y/o madre y la autopercepción corporal normal.

Palabras clave: Actividad Física; Sedentarismo; Adolescentes; Estado Nutricional; Sobrepeso; Obesidad.

1. Universidad CES, Medellín, Colombia.

2. Universidad Jaime I, Castellón de la plana, Comunidad Valenciana España.

Correspondencia: Carlos Augusto Poveda Acelas. Dirección: Calle 23 17-08 Bucaramanga. Teléfono: + 57 3184952932. Correo electrónico: carlos226_hotmail.com 


\begin{abstract}
Introduction: Excess weight in adolescents is one of the most frequent health problems worldwide, being attributed to social, family and lifestyle factors, which, if not modified, could generate physical, social, psychological and economic repercussions. Objective: To determine the relation between sociodemographic, physical activity and sedentary behaviors with the excess weight of adolescents (13 to 17 years old) in the Los Santanderes region of Colombia. Materials and methods: Analytical cross-sectional study, from a secondary source, from data from the National Survey of the Nutritional Situation in Colombia (Ensin 2015) on physical activity, sedentary lifestyle and its relationship with excess weight in adolescents teenagers in the region of Santanderes, Colombia. The study involved the performance of the chi-square hypothesis test with the calculation of OR as an epidemiological measure with their respective $95 \%$ confidence intervals. The multivariate analysis consisted of performing a binary logistic regression model for explanatory purposes. Results: No relationship was found between the social, family, body self-perception, physical activity and sedentary behavior variables with excess weight; however, the area of residence, particularly living in the municipal capitals (OR Crude: 1.,635, 95\% CI: 1.050 to 2.546) and selfperception of body weight as normal (Adjusted OR: 3.661, 95\% CI: 1.781a7.526) increase the chances of being overweight and obese. Conclusion: The implementation of interdisciplinary educational programs is required to prevent and treat excess weight in adolescents in a timely manner, taking into account that there are factors that show a certain relationship with excess weight such as living in municipal capitals, level of education of the head of household and/or mother and normal body self-perception.
\end{abstract}

Keywords: Exercise; Sedentary Behavior; Adolescent; Nutritional Status; Overweight; Obesity.

\section{Introducción}

El exceso de peso (sobrepeso y obesidad) en adolescentes es uno de los problemas de salud pública más prevalentes del siglo XXI, debido a que incrementa las probabilidades de presentar sobrepeso y obesidad en la edad adulta, convirtiéndose en factores de riesgo para el desarrollo de enfermedades crónicas ${ }^{1}$.

Diversos autores como Aguilar, et al. afirmaron que el número de adolescentes con sobrepeso y obesidad ha aumentado por 10 en el mundo en los cuatro últimos decenios, lo cual lo convierte en una epidemia global ${ }^{2}$, ya que según la Organización Mundial de la Salud (OMS), en 2016, había en el mundo más de 300 millones de niños y adolescentes con exceso de peso ${ }^{3}$.

Latinoamérica no es ajena a la problemática del exceso de peso en adolescentes. Autores como Sarmiento et al. afirmaron que el sobrepeso y obesidad en población infantil y adolescente es cada vez más preocupante ${ }^{4}$, por ejemplo, en países como Brasil, el exceso de peso pasó del $4,1 \%$ en 1974 a $22,2 \%$ en $2015^{5}$; así mismo, México ha venido presentando un aumento en los casos de sobrepeso y obesidad, donde el 17,9\% de los adolescentes con sobrepeso y el $9 \%$ con obesidad en 2006 pasó a $23,8 \%$ y $14,6 \%$ en 2018 , respectivamente ${ }^{6}$. Además, un estudio realizado en 2016 por Guedes, et al. en tres regiones latinoamericanas (Argentina, Brasil y Chile) reportó que entre 42,5 a 51,8 millones de jóvenes vivían con exceso de peso, lo cual representa aproximadamente el $20-25 \%$ del total de la población de adolescentes ${ }^{7}$.

En Colombia, la Encuesta Nacional de la Situación Nutricional (Ensin) reportó en 2010 de forma general que el $25,9 \%$ de los adolescentes presentaron exceso de peso; para 2015 la cifra del sobrepeso y obesidad fue de $17,9 \%$, dato que es mayor en el sector urbano que en el rural ${ }^{8}$.

Para la región Oriental que incluye a los Santanderes, lugar de interés de esta investigación, la Ensin 2010 y 2015 publicó que el $12,8 \%$ y 17,3\% de los adolescentes entre 13 y 17 años tenían sobrepeso y obesidad, respectivamente ${ }^{8}$.

Sumado a lo anterior, la literatura ha evidenciado que la inactividad física está vinculada con el exceso de peso: autores como Vinicio, et al. aluden a la falta de actividad física como una de las causas más importantes del sobrepeso y obesidad en los individuos 9 . De igual forma, datos a nivel mundial estiman que el $85 \%$ de las niñas y el $78 \%$ de los niños entre 11 a 17 años no cumplen con el mínimo de actividad física recomendado por la Organización Mundial de la Salud, quien especifica que esta práctica en los adolescentes debe tener una intensidad entre moderada a intensa y una 
hora diaria de duración. De esta manera, la inactividad física es uno de los factores de riesgo para el desarrollo de enfermedades crónicas, lo cual incrementa entre un 20 a $30 \%$ el riesgo de mortalidad en la población ${ }^{10}$.

En el panorama de Latinoamérica, las cifras de inactividad física y sedentarismo son desalentadoras. En México, por ejemplo, el $35 \%$ de los adolescentes no cumple con las recomendaciones de actividad física ${ }^{11}$; Brasil reporta que el $45 \%$ de los menores de edad son inactivos físicamente ${ }^{5}$.

En el caso de Colombia, según datos de la Ensin 2015, solo el $18,7 \%$ y $7,6 \%$ de los adolescentes cumplen las recomendaciones de actividad física, respectivamente; sumándose a esto, el 76,6\% de los menores de edad pasan un tiempo excesivo frente a las pantallas de televisores y videojuegos ${ }^{8}$.

De igual forma, en la literatura se ha encontrado que las influencias ambientales, principalmente el entorno familiar, estilos de vida inadecuados como la inactividad física, conductas sedentarias como jugar videojuegos y ver televisión en exceso han sido reportados por diversas investigaciones como factores de riesgo que pueden generar en los adolescentes sobrepeso y obesidad ${ }^{12}$.

Debido a lo anterior, este estudio buscó responder ¿cuál es la relación entre los factores sociales, de actividad física y comportamientos sedentarios con el exceso de peso de adolescentes de la región de los Santanderes, Colombia? El interés radica en la ausencia de estudios que aportaran información respecto a este tema en la región. En el mismo sentido, debido a que la adolescencia es una etapa de la vida donde se establecen conductas y hábitos que se mantendrán en la edad adulta.es clave promover estilos de vida saludables, con el fin de contribuir en la reducción del sobrepeso, obesidad y las enfermedades asociadas al exceso de peso $^{12}$, ya que como plantean Lee y Yoon en su investigación: la identificación y prevención tempranas son clave para controlar la epidemia mundial de obesidad en adolescentes ${ }^{13}$.

\section{Materiales y métodos}

Se trata de un estudio observacional analítico de corte transversal, de fuente de información secundaria, que buscó determinar la relación entre factores sociodemográficos, familiares, autopercepción corporal, actividad física y comportamientos sedentarios con el exceso de peso de los adolescentes (13 a 17 años) de la región de los Santanderes, Colombia.
La información de este estudio se obtuvo a través de la base de datos de la Ensin 2015, suministrada por el Ministerio de Salud y Protección Social, la cual contenía datos sociales, familiares, de autopercepción corporal, actividad física y comportamientos sedentarios de los adolescentes de la región de los Santanderes, Colombia.

El tipo de muestreo realizado para el estudio primario fue el probabilístico por conglomerados y polietápico, llevado a cabo en las zonas urbanas y rurales de los 1122 municipios de los 32 departamentos de Colombia ${ }^{14}$.

Para efectos de esta investigación, se tomó únicamente la información concerniente a los datos de los adolescentes de los departamentos de Santander y Norte de Santander (región los Santanderes), cuyo total fue de 847 registros.

Las variables independientes de este estudio fueron agrupadas en cuatro grupos: (i) sociodemográficos, (ii) sociales, (iii) de autopercepción corporal, (iv) actividad física y comportamientos sedentarios.

La Ensin 2015 empleó diversos instrumentos de recolección para las variables independientes que se tienen en cuenta en esta investigación. Para el grupo de variables sociodemográficas, la Ensin diseñó un cuestionario a través del cual se indagaron los datos sociales, área de residencia de los adolescentes, nivel de escolaridad, edad, sexo, etnia, afiliación al sistema de seguridad social, entre otros. Para evaluar la actividad física y los comportamientos sedentarios, la Ensin empleó el cuestionario del Youth Risk Behavior Surveillance System (YRBSS) ${ }^{14}$.

Una vez se tuvo la fuente de datos, se realizó la depuración de estos, y se eliminaron aquellas variables con subregistro mayor al $20 \%$. La variable dependiente de este estudio fue el estado nutricional, clasificado en dos niveles: con exceso de peso y sin exceso de peso; la categoría de interés de este estudio es el exceso de peso. Los menores que fueron asignados en los rangos con exceso de peso fueron aquellos menores de edad cuyo IMC/Edad se encontraba en sobrepeso $(>+1 \mathrm{a}+\leq 2$ $\mathrm{DE})$ y obesidad $(>+2 \mathrm{DE})$, mientras que en la categoría sin exceso de peso fueron situados los adolescentes con IMC/Edad adecuado ( $\geq-1 \mathrm{a} \leq+1 \mathrm{DE})$, riesgo de delgadez $(\geq-2 \mathrm{a} \leq-1 \mathrm{DE})$ y $\operatorname{delgadez}(<-2 \mathrm{DE})$. La clasificación del IMC/Edad fue realizada según la resolución 2465 de 2016 de Colombia, la cual establece los indicadores antropométricos, patrones de referencia y puntos de corte en Colombia para la clasificación 
del estado nutricional de niñas, niños y adolescentes menores de 18 años de edad, adultos de 18 a 64 años y gestantes adultas ${ }^{15}$.

En cuanto al grupo de variables independientes que conforman esta investigación son 46 y están distribuidas en 4 grupos: (i) sociodemográficas, (ii) sociales, (iii) autopercepción corporal, (iv) actividad física y comportamientos sedentarios. A continuación, se describen las variables que conforman cada grupo y las categorías asignadas.

Las variables sociodemográficas del estudio fueron: área de residencia (cabecera municipal, centro poblado, rural disperso); edad del adolescente (entre 13 a 15 años, entre 16 a 17 años); sexo del adolescente (masculino, femenino); nivel de escolaridad (menos de primaria completa, entre primaria completa y secundaria incompleta, entre secundaria completa y superior incompleta, superior completa); régimen de seguridad social (régimen contributivo o especial, régimen subsidiado, no afiliado).

El grupo de variables sociales lo conforman: sexo jefe del hogar (masculino, femenino); nivel de escolaridad del jefe del hogar (menos de primaria completa, entre primaria completa y secundaria incompleta, entre secundaria completa y superior incompleta, superior completa); actividad económica del jefe del hogar (desempleado, ocupado informal, ocupado formal); tipo de familia (nuclear, amplio, unipersonal-no familiar); nivel de escolaridad de la madre (menos de primaria completa, entre primaria completa y secundaria incompleta, entre secundaria completa y superior incompleta, superior completa).

Las variables del grupo de autopercepción y figura corporal fueron: percepción del peso corporal (normal, gordo, flaco); el adolescente está tratando de perder peso (sí, no) el adolescente está consumiendo menos grasa, harinas o dulces (sí, no); el adolescente está haciendo actividad física para perder peso (sí, no).

Finalmente, el grupo de variables de actividad física y comportamientos sedentarios está formado por: ejercicios de levantamiento de pesas en la semana ( 0 a la semana, 1 día a la semana, 2 días a la semana, 3 días a la semana, 4 días a la semana, 5 días a la semana, 6 días a la semana, 7 días a la semana); la mayoría de veces que fue al colegio lo hizo caminando, montando en bicicleta, en bus o transporte público, automóvil, motocicleta; tiempo que demoró en el recorrido de la casa al colegio (menos de 5 minutos, 5-15 minutos,
16-30 minutos, 31 minutos a 1 hora, más de una hora); el recorrido de regreso del colegio a la casa lo hizo caminando, montando bicicleta, en bus o transporte público, automóvil, motocicleta; tiempo que demoró en el recorrido de regreso del colegio a la casa (menos de 5 minutos, 5-15 minutos, 16-30 minutos, 31 minutos a 1 hora, más de una hora; presencia de centros deportivos en el barrio o vereda (sí, no); es seguro jugar en el parque o centro deportivo (sí, no); durante los últimos 12 meses algún médico le recomendó hacer actividad física (sí, no, no fue al médico en los últimos 12 meses); va a ciclovías los fines de semana (sí, no); el adolescente va a programas de clase dirigidas como aeróbicos, yoga (sí, no); programas de actividad física en el colegio (sí, no); asistencia a escuelas deportivas (sí, no); asistencia a grupos organizados de danzas o artes marciales (sí, no); horas TV entre semana (no vio TV en días escolares, menos de 1 hora, 1:00 a 1:59, 2:00 a 2:59, 3:00 a 3:59, 4:00 o más horas); horas que el adolescente juega videojuegos o usa el computador entre semana (no jugó ni uso el computador, menos de 1 hora, $1: 00$ a 1:59, 2:00 a 2:59, 3:00 a 3:59, 4:00 o más horas); horas que vio televisión durante el fin de semana (no vio televisión durante el fin de semana, menos de 1 hora, $1: 00$ a $1: 59,2: 00$ a 2:59, 3:00 a 3:59, 4:00 o más horas); horas que el adolescente usa computador o juega videojuegos en fin de semana (no jugó ni uso el computador, menos de 1 hora, 1:00-1:59, 2:002:59, 3:00-3:59, 4 o más); televisión en la alcoba del adolescente (sí, no); el adolescente tiene dispositivos electrónicos como PlayStation, Smartphone (sí, no).

Para el procesamiento de la información se utilizó el programa estadístico SPSS versión 26. El análisis univariado implicó el cálculo de frecuencias y porcentajes, dado que todas las variables eran de naturaleza cualitativa. Para el bivariado se elaboraron tablas de contingencia y la prueba de hipótesis chi cuadrado de independencia de Pearson con el cálculo de OR como medida epidemiológica, el cual se ajustó de acuerdo con las variables que tuvieron valores $\mathrm{p}<0,25$ en el análisis bivariado, dado que se puede hacer uso de esta prueba siempre y cuando se haga uso de la técnica de regresión logística. Finalmente, el análisis multivariado consistió en realizar un modelo de regresión logística binaria con fines explicativos (método intro), en donde ingresaron aquellas variables que tuvieron un valor $\mathrm{p}<0,25$, según criterio de Hosmer Lemeshow.

Para la realización de este estudio se tuvo en cuenta lo expuesto en la declaración de Helsinki y las pautas éticas de investigación en humanos; además, se contó 
con el permiso del Ministerio de Salud y Protección de Salud para el uso de la base de datos, el cual fue otorgado por correo electrónico; también se tuvo el aval del Comité de Ética de la Universidad CES Medellín Colombia.

\section{Resultados}

El estudio contó con 847 registros de los adolescentes de la región los Santanderes, Colombia, de los cuales 453 correspondían al sexo masculino y 394, al femenino. La prevalencia del exceso de peso fue de $20,4 \%(4,1 \%$ para la obesidad y $16,3 \%$ para el sobrepeso), lo cual equivale a 35 adolescentes con obesidad y 138 con sobrepeso.
Respecto a las características sociodemográficas se encontró que más del $56 \%$ de los adolescentes con exceso de peso tenían entre 13 a 15 años. En relación con el sexo, se encontró que poco más del $50 \%$ de los adolescentes con sobrepeso y obesidad eran de género masculino. En lo que atañe al área de residencia, más del $80 \%$ de los participantes de la investigación que presentaron exceso de peso vivían en cabeceras municipales. Aproximadamente el $50 \%$ de los sujetos del estudio con exceso de peso estaban afiliados al régimen subsidiado. En la Tabla 1 se puede observar que el área de residencia y régimen de seguridad social tuvieron un valor $\mathrm{p}<0,25$. Al analizar los OR crudos junto con los IC al $95 \%$, se puede aseverar que el vivir en la cabecera municipal aumenta las probabilidades de presentar sobrepeso y obesidad.

Tabla 1. Estado nutricional(sobrepeso/obesidad) según características sociodemográficas.

\begin{tabular}{|c|c|c|c|c|c|c|c|}
\hline \multirow{2}{*}{\multicolumn{2}{|c|}{ Variables Sociodemográficas }} & \multicolumn{6}{|c|}{ Estado nutricional (sobrepeso/obesidad) } \\
\hline & & \multicolumn{2}{|c|}{$\begin{array}{l}\text { Con exceso de } \\
\text { peso }\end{array}$} & \multicolumn{2}{|c|}{$\begin{array}{l}\text { Sin exceso de } \\
\text { peso }\end{array}$} & \multirow{2}{*}{ Valor P } & \multirow{2}{*}{ OR (IC 95\%) } \\
\hline & & $\mathbf{N}$ & $\%$ & $\mathbf{N}$ & $\%$ & & \\
\hline \multirow{3}{*}{ 1. Área de residencia } & Cabecera municipal & 139,0 & 80,3 & 495,0 & 73,4 & \multirow{3}{*}{0,06} & $1,6(1,05-2,54)$ \\
\hline & Centro poblado & 6,0 & 3,5 & 16,0 & 2,4 & & $2,18(0,8-6,0)$ \\
\hline & Rural disperso & 28,0 & 16,2 & 163,0 & 24,2 & & 1,00 \\
\hline \multirow{2}{*}{ 2. Edad del adolescente } & Entre 13 a 15 años & 98,0 & 56,6 & 394,0 & 58,5 & \multirow{2}{*}{0,66} & $0,92(0,66-1,30)$ \\
\hline & Entre 16 a 17 años & 75,0 & 43,4 & 280,0 & 41,5 & & 1,00 \\
\hline \multirow{2}{*}{ 3. Sexo del adolescente } & Masculino & 87,0 & 50,3 & 366,0 & 54,3 & \multirow{2}{*}{0,34} & $0,85(0,60-1,18)$ \\
\hline & Femenino & 86,0 & 49,7 & 308,0 & 45,7 & & 1,00 \\
\hline \multirow{3}{*}{$\begin{array}{l}\text { 4. Nivel de escolaridad } \\
\text { del adolescente }\end{array}$} & Menos de primaria completa & 9,0 & 5,2 & 22,0 & 3,3 & \multirow{3}{*}{0,44} & $1,44(0,57-3,64)$ \\
\hline & $\begin{array}{l}\text { Entre primaria completa y } \\
\text { secundaria incompleta }\end{array}$ & 145,0 & 83,8 & 581,0 & 86,7 & & $0,88(0,51-1,51)$ \\
\hline & $\begin{array}{l}\text { Entre secundaria completa y } \\
\text { superior incompleta }\end{array}$ & 19,0 & 11,0 & 67,0 & 10,0 & & 1,00 \\
\hline \multirow{3}{*}{$\begin{array}{l}\text { 5. Régimen de seguridad } \\
\text { social }\end{array}$} & Régimen contributivo o especial & 76,0 & 43,9 & 234,0 & 35,0 & \multirow{3}{*}{0,02} & 1,00 \\
\hline & Régimen subsidiado & 86,0 & 49,7 & 407,0 & 60,8 & & $0,65(0,45-0,91)$ \\
\hline & No afiliado & 11,0 & 6,4 & 28,0 & 4,2 & & $1,21(0,57-2,54)$ \\
\hline
\end{tabular}

En la Tabla 2 se muestran las características familiares de los participantes del estudio. Se puede afirmar que más del $60 \%$ de los padres de los menores de edad con exceso de peso tenían entre primaria completa y estudios universitarios incompletos. En cuanto al sexo del jefe del hogar, más del $50 \%$ era de sexo masculino. En lo concerniente a los resultados arrojados por la prueba de hipótesis de chi cuadrado, se evidencia que el nivel de escolaridad del jefe del hogar y de la madre de los menores de edad tuvieron asociación estadísticamente significativa con el exceso de peso.
En la Tabla 3 se observan las características de autopercepción y figura corporal, en donde más del $50 \%$ de los adolescentes con exceso de peso percibían que tenían sobrepeso y obesidad y, además, realizaban actividad física para perder peso. Los valores $\mathrm{p}$ de la prueba de chi cuadrado indican que todas las variables de este grupo se asociaron con el exceso de peso, excepto la variable actividad física para perder peso. Los OR crudos con los IC-95\% exponen que la percepción que tienen los adolescentes sobre su propio peso aumenta las probabilidades de padecer exceso de peso. 
Tabla 2. Estado nutricional (sobrepeso/obesidad) según variables familiares).

\begin{tabular}{|c|c|c|c|c|c|c|c|}
\hline \multirow{2}{*}{\multicolumn{2}{|c|}{ Variables Familiares }} & \multicolumn{6}{|c|}{ Estado nutricional (sobrepeso/obesidad) } \\
\hline & & \multicolumn{2}{|c|}{$\begin{array}{l}\text { Con exceso de } \\
\text { peso }\end{array}$} & \multicolumn{2}{|c|}{$\begin{array}{c}\text { Sin exceso de } \\
\text { peso }\end{array}$} & \multirow{2}{*}{ Valor $\mathbf{P}$} & \multirow{2}{*}{ OR (IC-95\%) } \\
\hline & & $\mathbf{N}$ & $\%$ & $\mathbf{N}$ & $\%$ & & \\
\hline \multirow{2}{*}{ 1. Sexo jefe del hogar } & Masculino & 99,0 & 57,2 & 415,0 & 61,6 & \multirow{2}{*}{0,29} & $0,83(0,59-1,17)$ \\
\hline & Femenino & 74,0 & 42,8 & 259,0 & 38,4 & & 1,00 \\
\hline \multirow{4}{*}{$\begin{array}{l}\text { 2. Nivel de escolaridad } \\
\text { del jefe del hogar }\end{array}$} & Menos de primaria completa & 45,0 & 26,0 & 179,0 & 26,7 & \multirow{4}{*}{0,04} & $0,48(0,24-0,96)$ \\
\hline & $\begin{array}{l}\text { Entre primaria completa y } \\
\text { secundaria incompleta }\end{array}$ & 53,0 & 30,6 & 256,0 & 38,2 & & $0,40(0,20-0,78)$ \\
\hline & $\begin{array}{l}\text { Entre secundaria completa y } \\
\text { superior incompleta }\end{array}$ & 59,0 & 34,1 & 204,0 & 30,4 & & $0,56(0,28-1,09)$ \\
\hline & Superior completa & 16,0 & 9,2 & 31,0 & 4,6 & & 1,00 \\
\hline \multirow{4}{*}{$\begin{array}{l}\text { 3. Actividad económica } \\
\text { del jefe del hogar }\end{array}$} & Desempleado & 1,0 & 0,7 & 8,0 & 1,6 & \multirow{3}{*}{0,50} & $0,41(0,05-3,39)$ \\
\hline & Ocupado informal & 71,0 & 51,8 & 282,0 & 55,6 & & $0,84(0,57-1,22)$ \\
\hline & Ocupado formal & 65,0 & 47,4 & 217,0 & 42,8 & & 1,00 \\
\hline & Nuclear & 100,0 & 57,8 & 380,0 & 56,4 & \multirow{3}{*}{0,79} & 1,00 \\
\hline \multirow[t]{2}{*}{ 4. Tipo familia } & Amplio & 72,0 & 41,6 & 292,0 & 43,3 & & $0,93(0,66-1,31)$ \\
\hline & Unipersonal- no familiar & 1,0 & 0,6 & 2,0 & 0,3 & & $1,90(0,17-21,16)$ \\
\hline \multirow{4}{*}{$\begin{array}{l}\text { 5. Nivel de escolaridad } \\
\text { de la madre }\end{array}$} & Menos de primaria completa & 23,0 & 16,1 & 139,0 & 24,6 & \multirow{4}{*}{0,01} & $0,36(0,16-0,81)$ \\
\hline & $\begin{array}{l}\text { Entre primaria completa y } \\
\text { secundaria incompleta }\end{array}$ & 39,0 & 27,3 & 188,0 & 33,3 & & $0,46(0,22-0,96)$ \\
\hline & $\begin{array}{l}\text { Entre secundaria completa y } \\
\text { superior incompleta }\end{array}$ & 68,0 & 47,6 & 209,0 & 37,0 & & $0,72(0,35-1,47)$ \\
\hline & Superior completa & 13,0 & 9,1 & 29,0 & 5,1 & & 1,00 \\
\hline
\end{tabular}

Tabla 3. Estado nutricional (sobrepeso/obesidad) según Autopercepción y figura corporal.

\begin{tabular}{|c|c|c|c|c|c|c|c|}
\hline \multirow{3}{*}{\multicolumn{2}{|c|}{ Variables Autopercepción y figura corporal }} & \multicolumn{6}{|c|}{ Estado nutricional (sobrepeso/obesidad) } \\
\hline & & \multicolumn{2}{|c|}{ Con exceso de peso } & \multicolumn{2}{|c|}{ Sin exceso de peso } & \multirow{2}{*}{ Valor P } & \multirow{2}{*}{ OR(IC-95\%) } \\
\hline & & $\mathbf{N}$ & $\%$ & $\mathbf{N}$ & $\%$ & & \\
\hline \multirow{3}{*}{ 1. Percepción del peso corporal } & Normal & 62,0 & 38,3 & 408,0 & 65,3 & \multirow{3}{*}{0,00} & $4,13(1,62-10,49)$ \\
\hline & Gordo & 95,0 & 58,6 & 81,0 & 13,0 & & $31,90(12,45-81,70)$ \\
\hline & Flaco & 5,0 & 3,1 & 136,0 & 21,8 & & 1,00 \\
\hline \multirow{2}{*}{$\begin{array}{l}\text { 2. El adolescente está tratando } \\
\text { de perder peso }\end{array}$} & $\mathrm{Si}$ & 91,0 & 56,2 & 76,0 & 12,2 & \multirow[b]{2}{*}{0,00} & 1,00 \\
\hline & No & 71,0 & 43,8 & 549,0 & 87,8 & & $0,10(0,07-0,16)$ \\
\hline \multirow{2}{*}{$\begin{array}{l}\text { 3. El adolescente está } \\
\text { consumiendo menos grasa, } \\
\text { harinas o dulces }\end{array}$} & $\mathrm{Si}$ & 91,0 & 74,0 & 108,0 & 60,3 & \multirow[b]{2}{*}{0,01} & 1,00 \\
\hline & No & 32,0 & 26,0 & 71,0 & 39,7 & & $0,53(0,32-0,88)$ \\
\hline \multirow{2}{*}{$\begin{array}{l}\text { 4. El adolescente está haciendo } \\
\text { actividad física para perder peso }\end{array}$} & $\mathrm{Si}$ & 68,0 & 55,3 & 101,0 & 56,4 & \multirow{2}{*}{0,84} & 1,00 \\
\hline & No & 55,0 & 44,7 & 78,0 & 43,6 & & $1,04(0,66-1,66)$ \\
\hline
\end{tabular}

La Tabla 4 describe las variables de actividad física y comportamientos sedentarios; se puede ver que poco más del $50 \%$ de los adolescentes con exceso de peso no realizaban ningún día a la semana ejercicios de fuerza, sin embargo, asistían a programas de clases dirigidas, escuelas deportivas y contaban con programas de actividad física en la escuela. Para las características de comportamientos sedentarios se observa que más del $65 \%$ de los menores de edad con exceso de peso tenían dispositivos electrónicos como Play Station, Smartphone y cerca del $47 \%$ de estos disponían de televisor en su habitación. En lo que respecta a los valores $\mathrm{p}$ de la prueba de chi cuadrado se evidencia que la "variable durante los últimos 12 meses algún médico le recomendó hacer actividad física" tuvo un valor $\mathrm{p}<0,05$. No obstante, los OR crudos y los intervalos de confianza no representan un aumento en las probabilidades de presentar exceso de peso. 
Relación entre actividad física, sedentarismo y exceso de peso en adolescentes de los Santanderes, Colombia

Tabla 4. Estado nutricional (sobrepeso/obesidad) según Actividad física y comportamientos sedentarios.

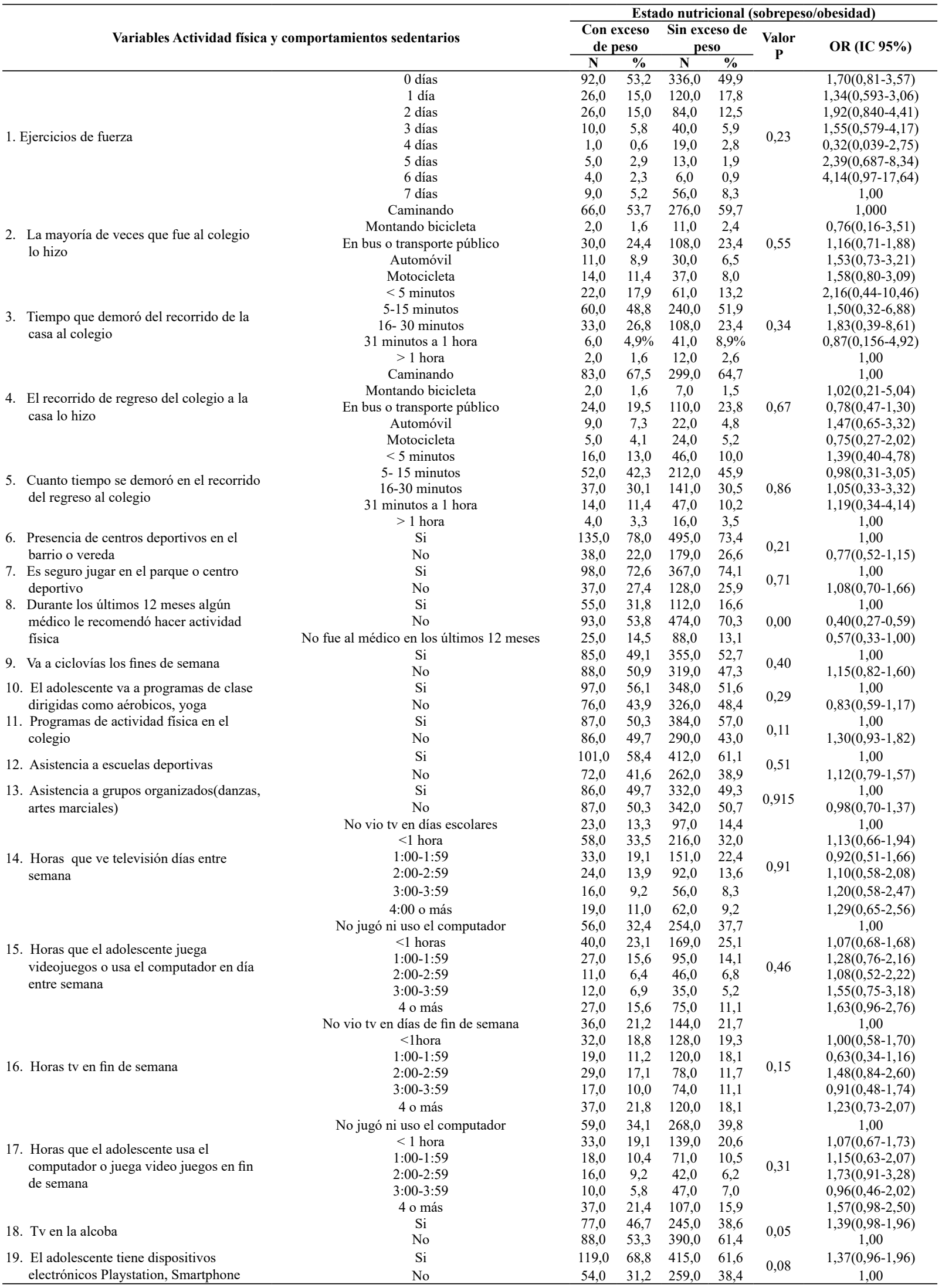


En la Tabla 5 se exponen las variables que ingresaron al modelo de regresión logística binaria. Al realizar una comparación entre OR crudos y OR ajustados se puede afirmar que ninguna de las variables evidenció cambios significativos. Finalmente, en lo concerniente a los OR ajustados junto con sus IC $-95 \%$, se puede observar que ninguno de los valores obtenidos indicó un aumento en la probabilidad de que los adolescentes presenten exceso de peso.

Tabla 5. Variables que explican la relación del exceso de peso.

\begin{tabular}{|c|c|c|c|c|}
\hline \multicolumn{2}{|c|}{ Variables que ingresaron al modelo } & \multirow{2}{*}{ Valor P } & \multirow{2}{*}{$\begin{array}{c}\text { OR Crudo IC-95\% } \\
1,63(1,05-2,54)\end{array}$} & \multirow{2}{*}{$\frac{\text { OR Ajustado IC } \mathbf{- 9 5} \%}{1,37(0,51-3,66)}$} \\
\hline & Cabecera municipal & & & \\
\hline 1. Área & Centro poblado & 0,06 & $2,18(0,78-6,05)$ & $2,11(0,25-17,38)$ \\
\hline \multirow{4}{*}{$\begin{array}{l}\text { 2. Afiliación al régimen de seguridad } \\
\text { social }\end{array}$} & Rural disperso & & 1,00 & 1,00 \\
\hline & Régimen contributivo o especial & \multirow{3}{*}{0,02} & 1,00 & 1,00 \\
\hline & Régimen subsidiado & & $0,65(0,45-0,91)$ & $0,55(0,25-1,22)$ \\
\hline & No afiliado & & $1,21(0,57-2,54)$ & $2,35(0,38-14,38)$ \\
\hline \multirow{4}{*}{ 3. Nivel de escolaridad jefe del hogar } & Menos de primaria completa & \multirow{4}{*}{0,04} & $0,48(0,24-0,96)$ & $0,64(0,09-4,42)$ \\
\hline & $\begin{array}{l}\text { Entre primaria completa y } \\
\text { secundaria incompleta }\end{array}$ & & $0,40(0,20-0,78)$ & $0,27(0,04-1,63)$ \\
\hline & $\begin{array}{l}\text { Entre secundaria completa y superior } \\
\text { incompleta }\end{array}$ & & $0,56(0,28-1,09)$ & $0,52(0,10-2,78)$ \\
\hline & Superior completa & & 1,00 & 1,00 \\
\hline \multirow{4}{*}{ 4. Nivel de escolaridad de la madre } & Menos de primaria completa & \multirow{3}{*}{0,01} & $0,36(0,16-0,81)$ & $0,63(0,10-4,01)$ \\
\hline & $\begin{array}{l}\text { Entre primaria completa y } \\
\text { secundaria incompleta }\end{array}$ & & $0,46(0,22-0,96)$ & $1,24(0,23-6,52)$ \\
\hline & $\begin{array}{l}\text { Entre secundaria completa y superior } \\
\text { incompleta }\end{array}$ & & $0,72(0,35-1,47)$ & $0,92(0,20-4,19)$ \\
\hline & Superior completa & & 1,00 & 1,00 \\
\hline \multirow{3}{*}{ 5. Percepción del peso corporal } & Normal & \multirow{3}{*}{0,00} & $4,13(1,62-10,49)$ & $3,661(1,78-7,52)$ \\
\hline & Gordo & & $31,90(12,45-81,70)$ & $0,30(0,02-3,53)$ \\
\hline & Flaco & & 1,00 & 1,00 \\
\hline \multirow{2}{*}{ 6. Pérdida de peso actualmente } & $\mathrm{Si}$ & \multirow{2}{*}{0,00} & 1,00 & 1,00 \\
\hline & No & & $0,10(0,07-0,16)$ & $0,36(0,17-0,78)$ \\
\hline \multirow{2}{*}{ 7. Alimentos no saludables y peso } & $\mathrm{Si}$ & \multirow{2}{*}{0,01} & 1,00 & 1,00 \\
\hline & No & & $0,53(0,32-0,88)$ & $0,68(0,32-1,45)$ \\
\hline \multirow{8}{*}{ 8. Ejercicios de fuerza } & 0 días & \multirow{8}{*}{0,23} & $1,70(0,81-3,57)$ & $1,92(0,60-6,09)$ \\
\hline & 1 días & & $1,34(0,59-3,06)$ & $1,30(0,35-4,78)$ \\
\hline & 2 días & & $1,92(0,84-4,41)$ & $5,19(1,37-19,68)$ \\
\hline & 3 días & & $1,55(0,57-4,17)$ & $0,87(0,17-4,45)$ \\
\hline & 4 días & & $0,32(0,03-2,75)$ & $2,12(0,15-28,83)$ \\
\hline & 5 días & & $2,39(0,68-8,34)$ & $0,88(0,00-2,33)$ \\
\hline & 6 días & & $4,14(0,97-17,64)$ & $4,21(0,41-42,63)$ \\
\hline & 7 días & & 1,00 & 1,00 \\
\hline \multirow{2}{*}{$\begin{array}{l}\text { 9. Presencia de centros deportivos en } \\
\text { el barrio o vereda }\end{array}$} & $\mathrm{Si}$ & \multirow{2}{*}{0,21} & 1,00 & 1,00 \\
\hline & No & & $0,77(0,52-1,15)$ & $0,82(0,35-1,88)$ \\
\hline \multirow{3}{*}{$\begin{array}{l}\text { 10. Durante los últimos } 12 \text { meses } \\
\text { algún médico le recomendó hacer } \\
\text { actividad física }\end{array}$} & $\mathrm{Si}$ & \multirow{3}{*}{0,00} & 1,00 & 1,00 \\
\hline & No & & $0,40(0,27-0,59)$ & $0,35(0,16-0,76)$ \\
\hline & $\begin{array}{l}\text { No fué al médico en los últimos } 12 \\
\text { meses }\end{array}$ & & $0,57(0,33-1,00)$ & $0,92(0,28-3,01)$ \\
\hline \multirow{2}{*}{$\begin{array}{l}\text { 11. Programa de actividad física en el } \\
\text { colegio }\end{array}$} & $\mathrm{Si}$ & 0.11 & 1,00 & 1,00 \\
\hline & No & & $1,30(0,93-1,82)$ & $1,42(0,70-2,87)$ \\
\hline & No vió tv en días de fin de semana & & 1,00 & 1,00 \\
\hline & $<1$ hora & & $1,00(0,58-1,70)$ & $0,56(0,20-1,56)$ \\
\hline & $1: 00-1: 59$ & & $0,63(0,34-1,16)$ & $0,67(0,20-2,29)$ \\
\hline 12. Horas tv en fin de semana & $2: 00-2: 59$ & 0,15 & $1,48(0,84-2,60)$ & $1,50(0,47-4,79)$ \\
\hline & $3: 00-3: 59$ & & $0,91(0,48-1,74)$ & $1,95(0,52-7,29)$ \\
\hline & 4 o más & & $1,23(0,73-2,07)$ & $0,59(0,21-1,61)$ \\
\hline 13. Ty en alcoba & $\mathrm{Si}$ & 0.05 & $1,39(0,98-1,96)$ & $1,29(0,63-2,63)$ \\
\hline 13. IV en alcoba & No & 0,05 & 1,00 & 1,00 \\
\hline 14. El adolescente tiene dispositivos & $\mathrm{Si}$ & & $1,37(0,96-1,96)$ & $1,64(0,79-3,39)$ \\
\hline $\begin{array}{l}\text { electrónicos play station, } \\
\text { Smartphone }\end{array}$ & No & 0,08 & 1,00 & 1,00 \\
\hline
\end{tabular}


Basado en lo anterior, se puede afirmar que no se encontró relación entre las variables analizadas en esta investigación y el exceso de peso. Sin embargo, el área de residencia, el nivel de escolaridad del jefe del hogar o de la madre y la percepción del peso corporal mostraron ser variables que pudieran ser desencadenantes del exceso de peso, en donde vivir en las cabeceras municipales y pobladas y la autopercepción que tenían los menores de edad acerca de su peso (percibirse con peso normal) pudieran provocar sobrepeso y obesidad en los adolescentes de la región.

\section{Discusión}

Este estudio permitió establecer la relación entre las variables sociodemográficas, familiares, de autopercepción, actividad física y comportamientos sedentarios con el exceso de peso, en donde el sobrepeso y la obesidad fue más prevalente en los adolescentes cuyas edades estaban entre los 13 a 15 años y pertenecían al género masculino; estos resultados coinciden con lo descrito por Muntaner, et al., quienes afirmaron que el exceso de peso era mayor en los adolescentes más jóvenes (10 a 12 años) y de género masculino ${ }^{16}$. Así mismo, fueron similares los hallazgos de Pinel, et al., cuya investigación arrojó que el porcentaje de exceso de peso fue mayor en los adolescentes varones, además no encontraron diferencias estadísticamente significativas con el sobrepeso y la obesidad coincidiendo con lo reportado por esta investigación ${ }^{17}$. Igualmente, García, et al. mostraron que la prevalencia del exceso de peso fue mayor en los adolescentes hombres $(26,1 \%)$ que en las mujeres $(20,6 \%)^{18}$. Cabe resaltar que el presente estudio pone de manifiesto que el efecto del sexo del adolescente sobre la probabilidad de sufrir de sobrepeso no se presenta en todos los contextos sociales y ambientales, pues para esta investigación no hubo una asociación significativa, tal como plantean Azar, et al. en su investigación ${ }^{19}$.

En lo concerniente al área de residencia, este estudio encontró que el vivir en las cabeceras municipales o zonas urbanas incrementa las probabilidades de padecer sobrepeso y obesidad, notando semejanzas con lo reportado por Shamah, et al. en donde el exceso de peso fue mayor en las zonas urbanas que en las rurales ${ }^{20}$. Análogos resultados encontraron Cambizaca, et al., quienes describieron que el sobrepeso $(20,54 \%$ en la zona urbana y $18,75 \%$ en el sector rural) y obesidad (7,03\% sector urbano y $3,13 \%$ área rural) era mayor en las zonas urbanas en comparación con las rurales ${ }^{21}$. Afines resultados halló Villena, quien descubrió que el exceso de peso estaba asociado a vivir en el área urbana $(\mathrm{OR}=1,9 \text {, IC 95\%:1,2 a 1,9 })^{22}$. Estos hallazgos podrían estar relacionados con la transición nutricional, en donde aquellos adolescentes que viven en el área urbana disminuyen la actividad física, tienen una alta frecuencia de horas frente al televisor que contribuyen significativamente a disminuir el gasto energético, además, emplean medios de transporte y generalmente tienen una ingesta energética más elevada. Lo anterior deja ver la importancia de crear intervenciones integrales que atiendan a las adolescentes desde edades más tempranas, donde se formen y fortalezcan hábitos saludables que prevengan el aumento excesivo de peso y se enfoquen en la atención y prevención ${ }^{23}$.

Respecto a las características familiares, esta investigación notó significancia estadística con el nivel de escolaridad del jefe del hogar y de la madre, parecido a lo descrito por Aristizábal, donde el nivel de escolaridad del tutor estaba asociada a la obesidad del adolescente $(\mathrm{OR}=0,44$ IC 95\%:0,02 A 0,94; valor P: 0,03$)^{24}$; de igual forma, Muntaner et al. y Campbell, et al. mostraron cómo el exceso de peso de los menores de edad estaba asociado al bajo nivel de escolaridad de la madre ${ }^{16,25}$, situación que confirma la influencia de los determinantes sociales sobre la salud y seguridad nutricional. El nivel educativo bajo puede influir en los estilos de vida, pues jefes de hogar y/o madres con menor nivel de escolaridad desconocen cómo debe ser la forma correcta de alimentación de un adolescente; además, la baja escolaridad se asocia con bajos ingresos económicos, lo cual podría indicar una dificultad para acceder a una alimentación balanceada con todos los grupos de alimentos que debe consumir un adolescente ${ }^{26}$.

Referente a las variables de autopercepción y figura corporal, el estudio arrojó que la percepción que tienen los adolescentes sobre su peso corporal junto con otros atributos como la pérdida de peso y la alimentación se encuentran vinculados al exceso de peso en los jóvenes. Lo anterior concuerda con lo expuesto por González, et al., donde la percepción del peso corporal que tenían los adolescentes (considerarse sin exceso de peso) fue estadísticamente significativa con el estado nutricional de estos $(p=<0,04)^{27}$; análogos hallazgos fueron los reportados por Ruiz, et al., quienes encontraron asociación entre la autopercepción corporal (percibirse con peso adecuado) y el índice de masa corporal $(p=0,01)^{28}$. El que los adolescentes se perciban con un peso normal cuando realmente están con exceso de peso es preocupante, ya que, al no percibirse con exceso de peso, es menos probable que realicen acciones para 
prevenirlo o tratarlo en los que casos que ya se tiene, pues para que una persona adopte una acción de salud, implica en primera instancia que se perciba vulnerable ${ }^{29}$. En cuanto a la alimentación se encontró similitud con lo concluido por García et al., quienes puntualizaron que los hábitos alimentarios no saludables estaban ligados al exceso de peso $(\mathrm{p}=<0,01)^{18}$.

En relación con las características de actividad física, en esta investigación se vio que más del $50 \%$ de los adolescentes con exceso de peso no realizaban ejercicios de fuerza como levantamiento de pesas. Sin embargo, realizaban otros tipos de actividad física como aérobicos o yoga, además que asistían a escuelas deportivas, encontrándose diferencias con lo planteado por Herazo, et al., quien reportó que más del $60 \%$ de los adolescentes con sobrepeso y obesidad no realizaban actividad física ${ }^{30}$. Así mismo, Cambizaca, et al. afirmaron que el $80 \%$ de los menores de edad con exceso de peso no hacían actividad física. La razón por la cual podrían diferir los resultados con esta investigación se debe a que en los estudios citados se midió la actividad física de manera general, mientras que en este estudio se indagaron diferentes aspectos en relación con el ejercicio físico ${ }^{21}$.

En lo que atañe a las variables de comportamientos sedentarios, en esta investigación no se halló asociación con el tiempo que pasan los adolescentes frente al televisor y computadores. Resultados afines explicaron García, et al., quienes no pudieron establecer asociación entre el tiempo frente a las pantallas y el IMC $(\mathrm{P}=0,224)^{18}$. Probablemente en este estudio no se encontró relación entre el exceso de peso y tiempo que pasan los adolescentes frente a pantallas, ya que como plantea Robinson, et al., en 2017, los resultados que no son estadísticamente significativos en estudios observacionales o transversales no indican que no exista una relación, pues este tipo de estudios es susceptible a errores en la medición del tiempo frente a la pantalla y, por consiguiente, tienen pérdida de poder estadístico. De manera que se sugiere estimar esta asociación por medio de estudios experimentales en los que se manipule la exposición a los medios de pantalla para probar la relación que existe ${ }^{31}$.

Para las variables televisión en la alcoba y dispositivos electrónicos, que obtuvieron valores $\mathrm{p}$ menor a 0,25 , autores como Heilmann, et al. afirmaron que tener este tipo de aparatos como la televisión en la alcoba estaba vinculado con el exceso de peso en los menores de edad ${ }^{32}$.
En relación con los aportes de esta investigación, se resalta que es la primera que indagó sobre la actividad física, comportamientos sedentarios y el exceso de peso en adolescentes de la región, cuyos resultados constituirán una guía para la creación de estrategias e intervenciones que permitan disminuir los efectos en el corto, mediano y largo plazo que llevan al sobrepeso y la obesidad.

Una de las limitaciones de este estudio fue la falta de variables sobre hábitos de consumo de alimentos, frecuencia de consumo de los alimentos y horas de sueño, los cuales en diversos estudios han mostrado la relación de estos elementos con el exceso de peso en los jóvenes.

Debido a lo anterior, se recomienda para futuras investigaciones indagar los aspectos en mención, con el fin de contar con datos claves que contribuyan a la formulación de políticas públicas dirigidas a los menores de edad con exceso de peso en la región, dadas las repercusiones físicas, psicológicas, económicas y sociales del sobrepeso y la obesidad.

\section{Conclusión}

La adolescencia es una etapa crucial en la adquisición de estilos de vida saludables que contribuirán en una mejora en la calidad de vida y por ende en la salud, por lo cual se hace necesario intervenir el problema del exceso de peso en los jóvenes con el fin de contrarrestar las consecuencias que este fenómeno produce. Para esto es importante identificar y realizar acciones de educación nutricional e intervenciones integrales que involucren las escuelas y familias, de manera que se pueda realizar prevención temprana y tratamiento de la obesidad y sobrepeso en aquellos adolescentes que tienen factores de riesgo asociados. Para efectos de esta investigación, los factores que tuvieron cierto vínculo con el sobrepeso y la obesidad fueron: el área de residencia, nivel de escolaridad de los padres y la autopercepción corporal, sin dejar de lado otros componentes sociales, familiares, de autopercepción, actividad física y comportamientos sedentarios que pudieran provocar sobrepeso y obesidad en la población juvenil.

\section{Agradecimientos}

Al Ministerio de Salud y Protección Social por facilitar la fuente de información. 


\section{Conflicto de interés}

Los autores declaramos no tener ningún conflicto de intereses.

\section{Referencias}

1. Morales A, Montilva M. Hábitos alimentarios no saludables, actividad física y obesidad en adolescentes entre 15 y 19 años. An Venez Nutr. 2016; 29(1): 4-10.

2. Aguilar C, Blanco L, Villareal E, Galicia L, Martínez L. Asociación de sobrepeso u obesidad con trastornos del estado de ánimo en adolescentes. Arc Latinoamericanos Nutrición. 2018; 68(4): 321-327.

3. Organización Mundial de la Salud. Obesidad y sobrepeso. Portal OMS; 2020. https://www.who. int/es/news-room/fact-sheets/detail/obesity-andoverweight

4. Sarmiento F, Ariza A, Barbosa F, Canal N, Benavides $\mathrm{M}$, Cruchet $\mathrm{S}$, et al. Sobrepeso y obesidad: revisión y puesta al día de la Sociedad Latinoamericana de Gastroenterología, Hepatología y Nutrición Pediátrica. Acta Gastroenterol Latinoam. 2016; 46(2): 131-159.

5. Lisbôa Conde W, da Silva Medeiros MC, Cumpian Silva J, Santana dos Santos IK dos Reis Santos AM. Estado nutricional de escolares adolescentes no Brasil: a Pesquisa Nacional de Saúde dos Escolares 2015. Rev Bras. Epidemiol. 2018; 21(Suppl 1): e180008. doi: https://doi.org/10.1590/1980549720180008.supl.1

6. Shamah T, Vielma E, Heredia O, Romero M, Mojica J, Cuevas L, et al. Encuesta Nacional de Salud y Nutrición 2018-19: Resultados Nacionales. Cuernavaca, México: Instituto Nacional de Salud Pública. (2020) https:/ensanut.insp.mx/encuestas/ ensanut2018/doctos/informes/ensanut_2018_ informe_final.pdf

7. Guedes DP, Villagra H, Moya J, del Campo J, Pirolli P, Pires R. Exceso de peso corporal y calidad de vida relacionada con la salud de adolescentes latino-americanos. Arch Med Deporte. 2017; 34(4): 201-206.

8. Instituto Colombiano Bienestar Familiar, Ministerio de protección social. Encuesta Nacional de Situación Nutricional. Portal ICBF - Instituto Colombiano de Bienestar Familiar ICBF; 2015. https://www. icbf.gov.co/bienestar/nutricion/encuesta-nacionalsituacion-nutricional
9. Vinicio M, Ramírez D. La promoción de la actividad física para disminuir el sobrepso en niños. Rev Podium. 2020; 15(1): 153-165. doi: https://doi. org $/ 10.2307 / j . c t v 105$ bcps

10. Organización Mundial de la Salud. New WHOled study says majority of adolescents worldwide are not sufficiently physically active, putting their current and future health at risk. Portal OMS; 2019.

11. Medina C, Jáuregui A, Campos I, Bárquera S. Prevalencia y tendencias de actividad física en niños y adolescentes: resultados de Ensanut 2012 y Ensanut MC 2016. Sal Pública Mex. 2018; 60(3): 263-271. doi: https://doi.org/10.21149/8819

12. Ruiz S, Mesquita M, Sánchez S. Actividad física y estado nutricional en adolescentes de 9 a 15 años de una institución de enseñanza de San Lorenzo, Paraguay. Pediatr (Asunción). 2017; 44(2): 111116. doi: https://doi.org/10.18004/ped.2017. agosto.111-116

13. Lee EY, Yoon, KH. Epidemic obesity in children and adolescents: risk factors and prevention. Front Med. 12; 6: 658-666. doi: https://doi.org/10.1007/ s11684-018- 0640-1

14. Encuesta Nacional de la Situación Nutricional en Colombia. Encuesta Nacional de la Situación Nutricional en Colombia-ENSIN. 2015; https://www.minsalud.gov.co/sites/rid/Lists/ BibliotecaDigital/RIDE/VS/ED/GCFI/documentometodologico-ensin-2015.pdf

15. Ministerio de Protección Social. Resolución 2465 de 2016. Minsalud; 2016. https://www.minsalud. gov.co/Normatividad_Nuevo/Forms/DispForm. aspx?ID=4909

16. Muntaner A, Conti J, Cantallops J, Borrás P, Palou P. Obesity and physical activity patterns among Balearic Islands children and adolescents: a crosssectional study. J Hum Sport Exercise. 2017; 12(1): 333-348. doi: https://doi.org/10.14198/ jhse.2017.122.10

17. Pinel C, Chacón R, Castro M, Espejo T, Zurita F, Pérez A. Diferencias de género en relación con el Índice de Masa Corporal, calidad de la dieta y actividades sedentarias en niños de 10 a 12 años. Retos. 2017; 31(1): 176-180. doi: https://doi. org/10.2307/j.ctvf3w3tj.25

18. García X, Allué N, Pérez A, Ariza C, Sánchez F, López $\mathrm{M}$, et al. Hábitos alimentarios, conductas sedentarias y sobrepeso y obesidad en adolescentes de Barcelona. An Pediatr (Barc). 2015; 83(1): 3-10. doi: https://doi.org/10.1016/j.anpedi.2014.07.006 
19. Azar A, Franetovic G, Martínez M, Santos Determinantes individuales, sociales y ambientales del sobrepeso y la obesidad adolescente en Chile. Rev Med Chile. 2015; 143: 598-605. doi: http:// dx.doi.org/10.4067/S0034-98872015000500007

20. Shamah T, Cuevas L, Gaona E, Gómez L, Morales M, Hernández M, et al. Sobrepeso y obesidad en niños y adolescentes en México, actualización de la Encuesta Nacional de Salud y Nutrición de Medio Camino 2016. Sal Pub México. 2018; 60(3): 244253. doi: https://doi.org/10.21149/8815

21. Cambizaca G, Castañeda I, Sanabria G, Morocho L. Factores que predisponen al sobrepeso y obesidad en estudiantes de colegios fiscales del Cantón Loja- Ecuador. Rev Habanera Ciencias Med. 2016; 15(2): 163-176. doi: https://doi.org/10.26820/ reciamuc/2.1.2018.480-490

22. Villena J. Prevalencia de sobrepeso y obesidad en el Perú. Rev Peru Ginecol Obstet. 2017; 63(4): 593598.

23. Medina M, Shamah T, Cuevas L, Méndez I, Hernández S. Factores de riesgo asociados con sobrepeso y obesidad en adolescentes mexicanas. Rev Salud Pública de México. 2020; 62: 125-136. doi: https://doi.org/10.21149/10388

24. Aristizábal P, García D, Vélez C. Determinantes sociales de la salud y su asociación con el sobrepeso y obesidad en adolescentes. Orinoquía. 2014; 18(2): 66-77. doi: https://doi.org/10.22579/20112629.301

25. Campbell M. Biological, environmental, and social influences on childhood obesity. Pediatric Research. 2016; 79(1): 205-211.

26. Barrera N, Fierro E, Puentes L, Ramos J. Prevalencia y determinantes sociales de malnutrición en menores de 5 años afiliados al Sistema de Selección de Beneficiarios para Programas Sociales (SISBEN) del área urbana del municipio de Palermo en Colombia, 2017. Univ Salud. 2018; 20(3): 236-246. doi: http://dx.doi.org/10.22267/rus.182003.126

27. González A. Insatisfacción de la imagen corporal y autopercepción en adolescentes de una escuela secundaria. Rev Neurol Neurocir Psiquiat. 2011; 44(4): 128-132.

28. Ruiz I, Carbonero R, Jáuregui I. Autopercepción del estado ponderal y la forma física y su relación con el nivel de actividad física realizado, conductas alimentarias y bienestar psicosocial. Nutr Hosp. 2015; 31(1): 203-216. doi: https://doi.org/10.24310/ riccafd.2018.v7i2.5098
29. Oliva-Peña Y, Ordóñez-Luna M, Santana-Carvajal A, Marín-Cárdenas AD, Andueza Pech G, Gómez Castillo IA. Concordancia del IMC y la percepción de la imagen corporal en adolescentes de una localidad suburbana de Yucatán. Rev Biomédica. 2016; 27(2): 49-60. doi: https://doi.org/10.32776/ revbiomed.v27i2.24

30. Herazo Y, Vidarte J, Sánchez L, Galeano L, Córdoba $\mathrm{J}$, Acuaña $\mathrm{G}$, et al. Nivel de actividad física e índice de masa corporal en escolares la región caribe colombiana:estudio multicéntrico. Rev Latino Hipertensión. 2019; 14(4): 225-231. doi: https://doi. org/10.18041/2390-0512/biociencias.2.6023

31. Robinson TN, Banda JA, Hale L, Lu AS, FlemingMilici F, Calvert SL, et al. Screen media exposure and obesity in children and adolescents. Pediatrics. 2017; 140(Suppl 2): S97-S101. doi: https://doi. org/10.1542/peds.2016-1758K

32. Heilmann A, Fitzsimons E, Kely Y, Watt R. Longitudinal associations between television in the bedroom and body fatness in a UK cohort study. International J Obesity. 2017; 41: 1502-1509. doi: https://doi.org/10.1038/ijo.2017.129 